\title{
Implications of the socio-physical contexts when interacting with mobile media
}

\author{
Anxo Cereijo Roibás · David Geerts · \\ Elizabeth Furtado · Licia Calvi
}

Published online: 6 March 2007

(C) Springer-Verlag London Limited 2007

\section{Introduction}

This Special Issue presents significant projects carried out in academia and in industry regarding the user experience with pervasive interactive audiovisual media systems such as mobile and pervasive iTV, cinema and video. Nomadic users such as commuters and travelers with purposes as diverse as entertainment, work and government are the main target users of these systems. In a nomadic life style, communication between individuals, groups and communities occurs over networks and devices that are most suitable for an individual's location, time, and context in general. So, special attention must be given to the social and contextual usage of these kinds of mobile applications.

The industry has often failed to identify the usage environments as well as to prospect users' real needs and expectations. Many companies have developed mobile devices and products or applications using inappropriate ICT resources that imply massive modifications in users' habits, thus resulting in perceptive and cognitive overload. Consequently, the market's response to investments in developing new products

A. C. Roibás $(\bowtie)$

SCMIS, University of Brighton, Brighton, UK

e-mail: anxo.cereijo@polimi.it; A.C.Roibas@bton.ac.uk

D. Geerts · L. Calvi

Centre for Usability Research (CUO), Katholieke

Universiteit Leuven, Leuven, Belgium

E. Furtado

University of Fortaleza, Fortaleza, Brazil

E. Furtado

Stanford University, Stanford, USA and applications (e.g. mobile TV broadcasting) has generally not been positive to date. Rapid changes in users' habits and technological advances have generated enormous uncertainties and call for innovative research and development methodologies. In fact, understanding user experiences that take place in different social contexts and physical and cultural environments is not an easy task for designers, who need to translate this information in design solutions.

A cross-disciplinary approach that includes human factor studies, behavioral theories, socio-cultural and economic trends, technological developments and emerging technologies markets, interactive arts, and product design is necessary to take into account the diverse nature of the problem of designing for pervasive interactive audiovisual media systems.

Moreover, several techniques such as collaborative and user-centered approaches that focus on users' cultural, social, behavioral and ergonomic backgrounds must be combined. As a matter of fact, most of the scenarios that companies create are technology driven, and the challenge is to create scenarios that are enduser-driven. These scenarios should not consider what users currently can do with the new technological possibilities, but they need to identify future needs and expectations, and find out how they can be served with the help of some new technologies and devices.

In social and contextual scenarios, successful examples of context aware applications show how users prefer methods of communication with capabilities that best meet a specific circumstance. For instance let us assume someone-interested in marine naturewhen passing by chance near an important whale watching spot along the coast, receives in his/her mobile a movie that someone else had recently 
recorded and consequently 'virtually uploaded' in the same place. It is clear how the contextualization of this experience might have a positive impact on the passerby who otherwise would miss the case. Furthermore, the mobile system can provide awareness about other people in the area with the same interests. ${ }^{1}$ In fact, the concept of context goes beyond the physical surroundings and includes the social environment. Consequently, designing for sociability is becoming a vital research area. In fact, nomadic communication contexts are characterized by strong collaboration and interaction needs between users. It is critical to identify suitable applications for the above scenarios as well as related new forms of content and novel interaction models that enable sociability within communities. This 'social' interaction is one of the challenges in designing pervasive interactive audiovisual media systems. Some basic underlying questions concern the possibilities for technology to support and encourage such communication and interaction among different users. In particular, they include questions about the kind of experience users expect to live by this sort of communication, the kind of information they are willing to disclose to their social relations, the kind of activities they require technology to support and the sort of interaction they ask the system to make possible.

Recurring themes in all articles of this special issue are the combination of different media, creating crossmedia experiences, social and collaborative aspects of audiovisual media, and the role of mobile devices in relation to audiovisual content.

In the first article, Primary and Secondary Context in Mobile Video Communication, Reponen, Huuskonen and Mihalic discuss the impact of the increasing use of video recording in public spaces. As more and more people carry public devices with them that allow for video recording, such as mobile phones, different situations arise in which people knowingly or not get captured on video that can be distributed in several ways via the internet. The authors use the concepts of primary context (immediate surroundings) and secondary context (published content) to describe the social impacts of using video recording in various situations. Variations in the primary context such as the visibility of the camera or the number of cameras have several implications regarding social or privacy con- cerns. In the secondary context, the timing of the distribution plays an important role. This article shows how new uses of audiovisual media devices create new situations that will have an important impact on our social and private lives.

Gislén, Löwgren and Myrestam describe in Avatopia: A Cross-Media Community for Societal Action how a cross-media platform for young teenagers was designed with the aim of influencing public opinion and changing society. From the project, the authors learned how a television broadcast to a large audience, showing creations that were made on a web community platform by a few community members, can create a positive spiral of production and consumption if tools with a strong audiovisual impact are used. They also describe how the participatory design process can strengthen a community because the involved teenagers feel a stronger commitment to the community, attracting and guiding new visitors in the community itself.

The third article, Modular Framework Support for Context-aware Mobile Cinema by Schrader, Carlson and Busch, deals with an artistic project where handheld computers are used to display interactive films based on the specific location and surroundings of the viewers. To allow for a flexible implementation without technical complexity for a broad range of devices and different environments, a Ubiquitous Computing Framework that supports context detection, context interpretation and mobile service delivery was developed.

Finally, in Interactive Movie Elements in a Pervasive Game, Ghellal and Lindt present a cross media multiplayer social adventure game involving live gameplay, video footage, a web interface and a broadcast television show. This interesting mix of several media brings up several research questions regarding, among others, virtual and physical experiences or social and collaborative aspects.

With increasing computing power also on mobile devices, and the familiarisation of wider audiences with more interactive television experiences, audiovisual media will play an important role in the future. The articles presented in this special issue give us an idea of how new concepts are being formed, making use of these new capabilities, and what social aspects are of importance in the new media landscape.

\footnotetext{
${ }^{1}$ Although privacy issues are crucial to this argument, they go beyond the scope of this Special issue.
} 